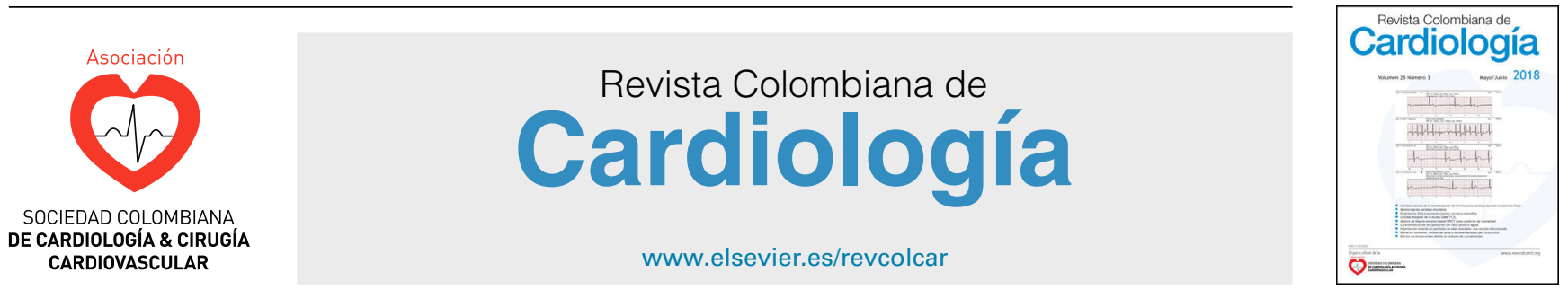

CARDIOLOGÍA DEL ADULTO - ARTÍCULO ORIGINAL

\title{
Síndrome vasomotor: puente de unión entre la disfunción endotelial y la esclerosis arterial
}

\section{Daysi Navarro Despaigne ${ }^{\mathrm{a}, *}$, Liudmila Staroushik Morel $^{\mathrm{a}}$ y Julio Oscar Cabrera Rego ${ }^{\mathrm{b}}$}

\author{
a Instituto Nacional de Endocrinología, La Habana, Cuba \\ ${ }^{\mathrm{b}}$ Hospital Docente Dr. Manuel Fajardo, La Habana, Cuba
}

Recibido el 12 de abril de 2017; aceptado el 13 de septiembre de 2018

Disponible en Internet el 3 de abril de 2019

\author{
PALABRAS CLAVE \\ Climaterio; \\ Disfunción endotelial; \\ Aterosclerosis \\ subclinica; \\ Síndrome vasomotor; \\ Aterosclerosis
}

\begin{abstract}
Resumen
Introducción: Los síntomas vasomotores y la disfunción endotelial probablemente resulten del déficit estrogénico postmenopausia; no obstante, la relación de ambos con la progresión de la aterosclerosis es un tema controversial.

Objetivo: Identificar en mujeres de edad mediana con disfunción endotelial, la presencia de aterosclerosis subclinica y de cambios cardiometabólicos durante la transición a la menopausia. Método: Estudio observacional de corte transversal, en el que se incluyeron 43 mujeres con edad entre 40-59 años, con disfunción endotelial previa y 14 mujeres con función endotelial normal. En cada mujer se identificaron: manifestaciones de rigidez arterial y del grosor de la grasa epicárdica y su relación con variables antropométricas, intensidad de los síntomas vasomotores, etapas del climaterio y niveles de estradiol.

Análisis estadístico: Regresión lineal múltiple y regresión logística para identificar asociación entre intensidad de los síntomas vasomotores, parámetros de rigidez arterial y cardiometabólicos $(\mathrm{p}<0,05)$ para significación estadística.

Resultados: Los parámetros de rigidez arterial (VLPP, CA, Al), de aterosclerosis (GIMC) y el grosor de la grasa epicárdica no mostraron cambios compatibles con daño arterial. A mayor intensidad de los síntomas vasomotores se encontró mayor probabilidad de incremento de la VLPP. Evolutivamente un subgrupo de mujeres desarrolló cambios metabólicos y disfunción endotelial sin asociación con el hipoestrogenismo, la edad ni la etapa del climaterio.

Conclusiones: Una mayor intensidad de los síntomas vasomotores podría constituir un marcador que identifique de manera temprana el riesgo de desarrollar aterosclerosis clínica.

(c) 2019 Sociedad Colombiana de Cardiología y Cirugía Cardiovascular. Publicado por Elsevier España, S.L.U. Este es un artículo Open Access bajo la licencia CC BY-NC-ND (http:// creativecommons.org/licenses/by-nc-nd/4.0/).
\end{abstract}

\footnotetext{
* Autor para correspondencia.

Correo electrónico: dnavarro@infomed.sld.cu (D. Navarro Despaigne).
} 


\section{KEYWORDS}

Menopause;

Endothelial

dysfunction;

Sub-clinical

atherosclerosis;

Vasomotor symptoms;

Atherosclerosis

\section{Vasomotor syndrome: meeting point between endothelial dysfunction and atherosclerosis}

\begin{abstract}
Introduction: Vasomotor symptoms and endothelial dysfunction probably result in a postmenopausal oestrogen deficiency. However, the relationship of both with the progression of atherosclerosis is a controversial subject.

Objective: To identify the presence of sub-clinical atherosclerosis in middle-aged women with endothelial dysfunction, as well as cardiometabolic changes during the transition to the menopause.

Method: A cross-sectional, observation study was conducted on 43 women between 40 and 59 years of age, with previous endothelial dysfunction, and 14 women with normal endothelial function. The variables recorded in each woman were, signs of arterial stiffening epicardial fat thickness and their relationship with anthropometric variables, intensity of the vasomotor symptoms, stages of menopause, and oestradiol levels.

Statistical analysis: Multiple linear regression and logistic regression was performed in order to identify an association between the intensity of the vasomotor symptoms, arterial stiffness, and cardiometabolic parameters. A $P<.05$ was significantly significant.

Results: The arterial stiffness parameters (pulse wave propagation (PWP), Arterial distensibility, augmentation index), and that of atherosclerosis (carotid intima-media thickness, GIMC), and the thickness of the epicardial fat showed no changes compatible with arterial damage. At a higher intensity of vasomotor symptoms, a higher probability of an increase in PWP was found. Evolutionarily, a sub-group of women developed metabolic changes and endothelial dysfunction with no relationship with low oestrogens, age, or the stage of the menopause.

Conclusions: A greater intensity of vasomotor symptoms could be a marker for the early identification of the risk for clinical atherosclerosis.

(c) 2019 Sociedad Colombiana de Cardiología y Cirugía Cardiovascular. Published by Elsevier España, S.L.U. This is an open access article under the CC BY-NC-ND license (http:// creativecommons.org/licenses/by-nc-nd/4.0/).
\end{abstract}

\section{Introducción}

Durante el período fértil la mujer está protegida contra la aterosclerosis, por tanto, en la posmenopausia la tasa de enfermedad de las arterias coronarias comienza a ascender exponencialmente, fenómeno que puede reflejar el impacto acumulativo de alteraciones tempranas producto de la presencia de factores de riesgo cardiovascular y de la pérdida de los efectos cardioprotectores de los estrógenos durante los primeros años de postmenopausia ${ }^{1-5}$.

En la práctica médica actual se dispone de un grupo de pruebas para el diagnóstico temprano de la aterosclerosis, entre las que pueden citarse la evaluación de la función endotelial, el score de calcio, la distensibilidad de las arterias y el engrosamiento de la íntima media en la carótida; al respecto se considera la disfunción endotelial como uno de los eventos iniciales en el desarrollo de la aterosclerosis, mientras que las otras técnicas expresan rigidez y daño arterial $^{6-10}$.

Durante la transición a la menopausia, diferentes investigadores reportan señales tempranas de aterosclerosis como disfunción endotelial, cambios en la distensibilidad arterial e incremento del grosor íntima-media de las arterias carótidas. Las dos primeras pueden ser modificadas por la terapia hormonal para la menopausia, y al grosor íntima media de las arterias carótidas se le señala valor predictivo para la estratificación del riesgo vascular incluso en individuos con bajo riesgo ${ }^{11-15}$; sin embargo, el puente de unión evolutivo/pronóstico entre eventos endoteliales y la rigidez arterial está aún por definirse.

La grasa epicárdica, como parte de la adiposidad visceral, se considera hoy día como un marcador emergente de riesgo cardiometabólico, y en población cubana se asocia con resistencia a la insulina, aterosclerosis subclínica, síndrome metabólico y relación ApoB/ApoA1 elevada, así como enfermedad arterial coronaria ${ }^{16-18}$.

Durante la perimenopausia y primeros años de postmenopausia, las oleadas de calor o síntomas vasomotores están entre las manifestaciones clínicas "típicas de la etapa" 19. En un estudio previo realizado en el Instituto Nacional de Endocrinología en mujeres aparentemente sanas en etapa de climaterio, se encontró un grupo con disfunción endotelial, en particular en aquellas con síntomas vasomotores de mayor intensidad y en la etapa temprana de la postmenopausia ${ }^{20}$, lo que motivó el interrogante siguiente: ¿constituye la intensidad del síntoma vasomotor un marcador inicial de aterosclerosis?

\section{Objetivo}

Identificar en mujeres de edad mediana con disfunción endotelial, la presencia de aterosclerosis subclínica y su relación 
con las etapas del climaterio, la intensidad de los síntomas vasomotores y los cambios cardiometabólicos.

\section{Métodos}

Estudio observacional, de corte transversal, que incluyó 43 mujeres aparentemente sanas con edad entre 40 a 59 años, con disfunción endotelial demostrada mediante dilatación mediada por flujo de la arteria braquial, y 14 mujeres aparentemente sanas sin disfunción endotelial, entre 2012 a 2015.

\section{Procedimiento}

Mediante llamado telefónico o directamente se citaron al servicio de Ecocardiografía del Hospital "Manuel Fajardo" 52 mujeres sin factores de riesgo cardiovascular con disfunción endotelial diagnosticadas previamente. Respondieron 43 mujeres (83\% las causas de no respuesta: salidas del país y problemas personales). Después de obtener su consentimiento, a cada una se le realizó:

- Extracción de sangre en ayunas para determinaciones bioquímicas y hormonales).

- Ultrasonido carotídeo.

- Ecocardiograma.

- Evaluación realizada entre 3 y 6 meses del diagnóstico de la disfunción endotelial.

- Un año después fueron citadas aquellas sin disfunción endotelial previa, de las cuales respondieron 14 de 30 a las que se les hizo reevaluación clínica, bioquímica, hormonal y endotelial, además de identificar manifestaciones de rigidez arterial.

\section{Variables clínicas}

Edad actual, frecuencia cardiaca, presión arterial sistólica [PAS] y diastólica [PAD], circunferencia de cintura, índice de masa corporal, etapas del climaterio e intensidad del síndrome vasomotor.

\section{Variables bioquímicas}

Glucemia de ayunas, colesterol total y triglicéridos.

\section{Variables hormonales}

Estradiol y FSH.

\section{Variables ecocardiográficas}

Grosor íntima grosor íntima media de las arterias carótidas. Parámetros de rigidez arterial (velocidad local de propagación del pulso carotídeo [VLPP], elastancia [Ep], compliance arterial [CA] e índice de rigidez arterial $\beta$ ).

Grosor de la grasa epicárdica.

\section{Definiciones}

Según etapas del climaterio, las mujeres se clasificaron como peri o posmenopausia y esta última como etapa temprana (referida a los cinco primeros años de posmenopausia) y etapa tardía (desde cinco años después de la menopausia hasta los 64 años).

Los síntomas vasomotores se clasificaron como muy leve, leve, moderado y severo o crítico según valores obtenidos mediante la escala de Pérez Piñero ${ }^{21}$ con base en los siguientes parámetros: muy leve: 0- 6 puntos; leve 7-14 puntos; moderado 15-23 puntos y severo o crítico 24 puntos o más.

La circunferencia de la cintura se determinó al medir el perímetro abdominal a nivel del punto medio entre el último arco costal y la cresta ilíaca antero superior con la paciente previamente desvestida. El índice de masa corporal se calculó según la fórmula de Quetelet: peso en kg/ (talla en $\mathrm{m}^{2}$ ).

\section{Parámetros bioquímicos}

Para la glucemia, el colesterol total y los triglicéridos se emplearon los reactivos RapiGluco-Test, Colestest y Monotriglitest, respectivamente, producidos en el EPB "Carlos J. Finlay", La Habana, Cuba. La glucemia fue procesada en un equipo Eppendorf mediante método enzimático colorimétrico y el colesterol y los triglicéridos en un equipo Hitachi 7170 A, Tokio, Japón. Los resultados se reportaron de acuerdo con el Sistema Internacional de Unidades.

\section{Determinaciones hormonales}

La FSH (hormona folículo estimulante): se determinó en etapa folicular en las mujeres que menstrúan y en cualquier momento en las que estaban en amenorrea. Se determinó mediante el principio del análisis inmunorradiométrico (IRMA) utilizando los kits disponibles en el Instituto Nacional de Endocrinología. Su concentración sérica de referencia es de 0,6-9,5 Ul/ml en fase folicular de las mujeres y en las mujeres postmenopáusicas de $30-135 \mathrm{mUl} / \mathrm{ml}$. El estradiol se determinó en etapa folicular (entre el tercero y quinto día del ciclo menstrual) en las mujeres que menstrúan y en cualquier momento en aquellas en amenorrea, mediante el inmunoensayo ECLIA (Electro chemiluminiscence inmuno assay) utilizando el equipo inmunoanalizador Cobas 411. Valores de referencia en fase folicular $(46,0-607 \mathrm{pmol} / \mathrm{l}) \mathrm{y}$ en mujeres posmenopáusicas $(18,4-201 \mathrm{pmol} / \mathrm{l})^{22}$.

\section{Parámetros del ecocardiograma}

Previo a su realización después de llegar la paciente al departamento se determinaron las PAS y PAD con esfigmomanómetro de mercurio; posteriormente, mediante equipo de ultrasonidos (Aloka, Alfa-10, MitakaShi, Tokio, Japón), se precisó:

\section{- Grosor íntima media carotídeo.}

Parámetros de rigidez arterial: el programa determinó automáticamente los valores de la velocidad de la onda del 
pulso que transita por la arteria carótida (VLPP), de la presión que ejerce la sangre para deformar el vaso (Ep), del cambio de volumen por la presión sanguínea (CA) y del índice de rigidez arterial $\beta$ (cambio del diámetro en sístole de una arteria respecto a la diástole durante el ciclo cardíaco).

Grosor grasa epicárdica, para lo cual: se colocó el paciente en decúbito supino con ligera laterización del cuello, ubicándose un transductor lineal con frecuencia de $7,5 \mathrm{MHz}$ en posición longitudinal respecto a la arteria carótida común (posición lateral superior del cuello por dentro del músculo esternocleidomastoideo), con la señal del electro-cardiograma activada para obtener imágenes vasculares al final de la diástole, visualizándose con una profundidad de $4 \mathrm{~cm}$. Se examinaron ambas arterias carótidas comunes en un segmento de $10 \mathrm{~mm}$ distal a la aparición del bulbo carotídeo. Se hicieron las mediciones a nivel de la pared posterior, con un programa semiautomático de detección de bordes. Los percentiles carotídeos de la arteria carótida común derecha e izquierda se determinaron de acuerdo con edad, sexo y raza del paciente. Se consideró grosor íntima media de las arterias carótidas aumentado a valores por encima del percentil 75 para edad, sexo y raza ${ }^{23}$. Los parámetros de rigidez arterial se obtuvieron según software del equipo. Se realizaron tres mediciones y se tomó el valor promedio. En población cubana sin hipertensión arterial los valores de referencia de la VLPP son de 4,7 $\mathrm{m} / \mathrm{s}^{24}$.

La grasa epicárdica se determinó en las vistas eje largo paraesternal tomando como referencia de corte el plano valvular aórtico y el eje corto paraesternal a nivel de los músculos papilares. Para la determinación de la grasa epicárdica anterior al ventrículo derecho se promediaron los valores obtenidos de las vistas eje largo y eje corto paraesternal. La grasa epicárdica se midió en el período telesistólico del ciclo cardiaco. En mujeres adultas cubanas aparentemente sanas, los valores de referencia son menores a $4,9 \mathrm{~mm}^{24}$.

\section{Análisis estadístico}

Se aplicó el programa SPSS versión 18.0 (SPSS Inc., Chicago, III, USA). Las variables cuantitativas se expresaron como mediana y rango. Se aplicó un análisis univariado mediante la prueba de probabilidad exacta de Fisher para las variables cualitativas y la prueba $U$ de Mann-Whitney para las variables cuantitativas. Se estableció un valor bilateral de $p<0,05$ para definir la significancia estadística y se utilizó ANOVA para establecer diferencias en más de dos grupos.

Mediante regresión lineal múltiple se identificó la influencia de los niveles de estradiol con las variables clínicas, bioquímicas y ultrasonográficas, así como la velocidad del pulso carotídeo (VLPP) con las variables clínicas y bioquímicas.

Mediante regresión logística para identificar la influencia de la intensidad de los síntomas vasomotores sobre el grosor íntima media de las arterias carótidas, VLPP y grasa epicárdica, se utilizó valor de $p<0,05$ para la significación estadística.

\section{Aspectos éticos}

El reclutamiento de las pacientes se hizo mediante consentimiento informado. La participación fue totalmente voluntaria y la no participación no afectó la atención por el médico de la familia. Se garantizó la confidencialidad de los datos que se obtuvieron. El personal que llevó a cabo la investigación contó con la preparación necesaria para realizarla. En los casos en que se detectaron alteraciones en el examen físico y/o los complementarios, que sugerían enfermedad, se garantizó la atención médica correspondiente.

\section{Resultados}

El grupo total estuvo constituido por 57 mujeres: 43 con disfunción endotelial y 14 sin disfunción endotelial previa.

En la tabla 1 se resumen las principales características clínicas, hormonales, bioquímicas y variables del ecocardiograma según etapa del climaterio del grupo de mujeres con disfunción endotelial previa; todos los parámetros evaluados estuvieron en al rango de referencia.

En relación con el grosor íntima media de las arterias carótidas y demás elementos de rigidez arterial, ninguna de las variables presentó valores compatibles con daño arterial; no obstante, hubo tendencia a la rigidez arterial (mayor VLPP, menor compliance y mayor IA) en las mujeres en etapa tardía de la postmenopausia (tabla 2).

Según la intensidad de los síntomas vasomotores no se encontraron diferencias entre los valores de la mediana de las variables del ecocardiograma. Se identificó correlación positiva entre la velocidad del pulso carotídeo (VLPP) como expresión de rigidez arterial con la edad y el grosor de la grasa epicárdica y entre esta última con el índice de masa corporal y con la VLPP (tabla 3).

El modelo de regresión lineal múltiple asumiendo la VLPP como variable dependiente no mostró asociación significativa con las variables clínicas ni hormonales (tabla 4). Mediante regresión logística asumiendo los síntomas vasomotores dicotómicos como de leves e intensos se encontró que la intensidad incrementa seis veces el riesgo de aumentar la VLPP (tabla 5).

Catorce mujeres sin disfunción endotelial previa fueron reevaluadas un año después ( $X 14$ meses) y se encontró que 4 de ellas tuvieron disfunción endotelial. En la tabla 6 se muestran las diferencias clínicas, bioquímicas y hormonales entre ambos subgrupos (tabla 6).

No hubo diferencias entre los valores del grosor íntima media de las arterias carótidas $(0,70 \mathrm{vs} .0,76 \mathrm{~mm})$ y del grosor de la grasa epicárdica $(5,5 \mathrm{vs}$. $5,5 \mathrm{~mm})$ entre el grupo de mujeres que se mantuvieron sin disfunción endotelial respecto al grupo que desarrolló la misma.

\section{Discusión}

La enfermedad cardiovascular es consecuencia de un proceso continuo que se inicia desde la concepción y se desarrolla como resultado de múltiples factores, entre los que se citan la hipertensión arterial, la diabetes mellitus, 
Tabla 1 Características clínicas, hormonales, bioquímicas y ecocardiograficas en mujeres con disfunción endotelial

\begin{tabular}{|c|c|c|c|c|}
\hline Variables & Perimenopausia $n=11$ & Postetapa temprana $n=15$ & Postetapa tardía $n=16$ & Valor $\mathrm{p}$ \\
\hline \multicolumn{5}{|l|}{ Variables clínicas } \\
\hline Edad (años) & $48\left(\begin{array}{ll}1 & 3\end{array}\right)$ & $50(12)$ & $56(8)$ & 0,00 \\
\hline IMC (kg/talla m²) & $24,1(7,8)$ & $24,8(9,1)$ & $26,2(7,8)$ & 0,084 \\
\hline $\mathrm{CC}(\mathrm{cm})$ & $89,0(23,5)$ & $82,0(37)$ & $88,5(38)$ & 0,242 \\
\hline PAS mm Hg) & $110(20)$ & $120(40)$ & $120(30)$ & 0,347 \\
\hline PAD mm Hg & $60(20)$ & $80(20)$ & $80(10)$ & 0,044 \\
\hline FC latidos/min & $70(11)$ & $67(25)$ & $78(29)$ & 0,303 \\
\hline \multicolumn{5}{|l|}{ Variables hormonales } \\
\hline FSH & $43,48(127,41)$ & $61,90(75,29)$ & $69,07(74,68)$ & 0,128 \\
\hline Estradiol & $145,82(220,95)$ & $30,12(41,19)$ & $25,33(3,21)$ & 0,136 \\
\hline \multicolumn{5}{|l|}{ Variables bioquímicas } \\
\hline Glucemia (mmol/L) & $4,7(3,0)$ & $4,3(, 49)$ & $4,4(1,3)$ & 0,094 \\
\hline Colesterol total (mmol/L) & $5,5(1,0)$ & $5(, 4,6)$ & $5(3,1)$ & $0,039^{*}$ \\
\hline Triglicéridos (mmol/L) & $1,45(1,6)$ & $1,1(0,33)$ & $1,1(0,5)$ & 0,607 \\
\hline \multicolumn{5}{|l|}{ Variables ecocardiográficas } \\
\hline GIM & $0,590(0,260)$ & $0,625(0,23)$ & $0,645(0,6)$ & 0,431 \\
\hline VLPP & $4,55(2,15)$ & $4,700(1,95)$ & $5,500(1,4)$ & 0,000 \\
\hline Ep & $53,55(43,00)$ & $54,500(52,00)$ & $83,000(54,5)$ & 0,000 \\
\hline CA & $0,755(0,83)$ & $0,830(0,96)$ & $0,565(0,9)$ & 0,010 \\
\hline $\mathrm{Al}$ & $13,70(43,9)$ & $12,55(32,8)$ & $24,07(29,7)$ & 0,146 \\
\hline Grasa epicárdica & $2,900(4,5)$ & $3,600(6,3)$ & $4,700(3,9)$ & 0,002 \\
\hline
\end{tabular}

Al: índice de rigidez arterial; CA: complianza arterial; CC: circunferencia de cintura; Ep: constante de elastancia (presión/deformación); FC: frecuencia cardiaca; FSH: hormona folículo estimulante; GIM: grosor intima media; IMC: índice de masa corporal; PAD: presión arterial diastólica; PAS: presión arterial sistólica; VLPP: velocidad local de propagación del pulso.

Valores expresados como mediana (rango)

Con relación a las variables clínicas se observa mayor edad entre las mujeres con mayor tiempo de posmenopausia, grupo en que además se reportan los menores valores de estradiol y en que los parámetros del ecocardiograma mostraron tendencia a la rigidez arterial (mayores valores del VLPP, del Ep del Al).

Tabla 2 Intensidad de síntomas vasomotores y variables ecocardiográficas en mujeres con disfunción endotelial

\begin{tabular}{llllll}
\hline Variable & Asintomátican $=9$ & Ligeros $n=12$ & Moderados $n=7$ & Severos $n=15$ & Valor $p$ \\
\hline GIM $(\mathrm{mm})$ & $0,770(0,260)$ & $0,590(0,130)$ & $0,720(0,100)$ & $0,620(0,660)$ & 0,022 \\
VLPP $(\mathrm{m} / \mathrm{s})$ & $4,700(1,170)$ & $5,100(2,300)$ & $4,700(1,550)$ & $5,200(2,150)$ & 0,561 \\
Ep $(\mathrm{kPa})$ & $58,000(25,000)$ & $65,000(63,000)$ & $58,000(44,000)$ & $60,500(55,500)$ & 0,912 \\
$\mathrm{CA}\left(\mathrm{mm}^{2} / \mathrm{kPa}\right)$ & $0,650(0,300)$ & $0,635(0,695)$ & $0,830(0,700)$ & $0,750(0,960)$ & 0,638 \\
$\mathrm{Al}(\%)$ & $21,100(32,850)$ & $14,200(35,650)$ & $24,100(23,950)$ & $17,875(43,950)$ & 0,990 \\
Grasa epicárdica $(\mathrm{mm})$ & $3,00(4,9)$ & $3,650(4,0)$ & $4,20(5,0)$ & $4,30(5,5)$ & 0,378 \\
\hline
\end{tabular}

Al: índice de rigidez arterial; CA: complianza arterial; Ep: constante de elastancia (presión/deformación); GIM: grosor intima media; VLPP: velocidad local de propagación del pulso.

No hubo diferencias significativas entre los valores de la mediana de parámetros del ecocardiograma y la intensidad de los síntomas vasomotores.

el incremento de la edad y el sexo. En relación con este último se conoce el factor protector de los estrógenos y las consecuencias que para el endotelio vascular tiene el déficit estrogénico posterior a la menopausia, al originar un desequilibrio en la biodisponibilidad de sustancias activas de origen endotelial, que predispone a la inflamación, vasoconstricción e incremento de la permeabilidad vascular (disfunción endotelial), lo que se considera como el evento inicial y por tanto más precoz de la aterosclerosis ${ }^{2,19}$.

Sariol y Navarro ${ }^{20}$ en mujeres aparentemente sanas (con bajo riesgo cardiovascular), en etapa de climaterio, encontraron disfunción endotelial en el $38 \%$, siendo más frecuente en la perimenopausia y en mujeres con síntomas vasomotores de mayor intensidad o grado de molestia y consideraron que tal vez esta expresión clínica podría "marcar" tanto las manifestaciones clínicas de la transición a la menopausia como la presencia de daño endotelial.

Los métodos para identificar el desarrollo del proceso de aterosclerosis permiten evaluar la aterosis, es decir, los cambios modificables en la íntima y la media de la pared vascular (disfunción endotelial), y por otra la esclerosis, o los cambios establecidos en la pared vascular, lo que justifica en la fisiopatogenia de la aterosclerosis que el camino 
Tabla 3 Correlación entre variables de rigidez arterial (VLPP) y de riesgo cardiometabólico (grasa epicárdica) con variables clínicas

\begin{tabular}{|c|c|c|}
\hline Variables control & Correlación de Pearson & Valor $\mathrm{p}$ \\
\hline \multicolumn{3}{|c|}{ VLPP variable dependiente expresión rigidez arterial } \\
\hline Edad & 0,423 & $0,05^{*}$ \\
\hline IMC & 0,139 & 0,381 \\
\hline $\mathrm{CC}$ & 0,160 & 0,922 \\
\hline Estradiol & $-0,483$ & 0,14 \\
\hline FSH & 0,133 & 0,415 \\
\hline Grasa epicárdica & 0,421 & $0,05^{*}$ \\
\hline \multicolumn{3}{|c|}{ Grosor grasa epicárdica variable dependiente } \\
\hline Edad & 0,265 & 0,086 \\
\hline IMC & 0,443 & $0,003^{*}$ \\
\hline $\mathrm{CC}$ & 0,467 & 0,620 \\
\hline Estradiol & 0,269 & 0,184 \\
\hline FSH & 0,133 & 0,415 \\
\hline VLPP & 0,421 & $0,005^{*}$ \\
\hline
\end{tabular}

CC: circunferencia de cintura; FSH: hormona folículo estimulante; IMC: índice de masa corporal; VLPP: velocidad local de propagación del pulso.

Describe resultados del modelo de correlación lineal. Nótese asociación significativa entre la VLPP con la edad y la grasa epicárdica y entre esta última con el índice de masa corporal y la VLPP.

*Asociación significativa entre las variables.

Tabla 4 Modelo de regresión lineal múltiple entre algunas variables clínicas y la velocidad de propagación del pulso

\begin{tabular}{|c|c|c|c|c|c|c|}
\hline \multirow[t]{2}{*}{ Modelo } & \multicolumn{2}{|c|}{ Coeficientes no estandarizados } & \multirow[b]{2}{*}{$\mathrm{t}$} & \multirow[b]{2}{*}{ Sig. } & \multicolumn{2}{|c|}{ Intervalo de confianza de $95,0 \%$ para B } \\
\hline & B & Error típico & & & Límite inferior & Límite superior \\
\hline (Constante) & 2,725 & 3,739 & & & $-5,245$ & 10,694 \\
\hline Edad &,- 014 & ,072 &, 729 & ,477 &,- 167 & ,139 \\
\hline IMC &,- 074 & ,141 &,- 196 & ,847 &,- 375 & ,227 \\
\hline CCin & 031 & ,060 &,- 525 & 607 &,- 097 & ,159 \\
\hline PAS & 012 & ,013 & ,522 & 610 &,- 016 & ,040 \\
\hline Etapa climatérica & 1,059 & ,454 & ,931 & ,367 & ,092 & 2,026 \\
\hline FSH &,- 009 & ,006 & 2,334 & ,034 &,- 023 & ,004 \\
\hline Estradiol &,- 003 & ,003 & $-1,518$ & , 150 &,- 009 & ,003 \\
\hline Grasa epicárdica &,- 061 & ,184 & $-1,135$ & ,274 &,- 453 & ,331 \\
\hline
\end{tabular}

Variable dependiente VLPP

PAS: presión arterial sistólica; IMC: índice de masa corporal; CCin: circunferencia de la cintura; FSH: hormona folículo estimulante. No se demuestra asociación significativa entre la VLPP con las variables evaluadas.

Tabla 5 Regresión logística entre intensidad de los síntomas vasomotores y variables del ecocardiograma

\begin{tabular}{lllll}
\hline SMV & OR & Std. Err. & z & P> [z] \\
\hline Grosor íntima media de las arterias carótidas & 0,644589 & 0,4915479 & $-0,58$ & 0,565 \\
VLPP & 6,266335 & 5,4191115 & 2,12 & $0,034^{*}$ \\
Grasa epicárdica & 1,704787 & 1,350827 & 0,67 & 0,501 \\
Constante &, 1462528 &, 1148637 & $-2,45$ & 0,014 \\
\hline
\end{tabular}

SMV: síndrome vasomotor (variable dependiente); VLPP: velocidad local de propagación del pulso carotídeo; grosor íntima media de las arterias carótidas: grosor íntima-medio carotídeo.

La intensidad de los síntomas vasomotores mostró incremento significativo del riesgo para aumento de la velocidad de propagación del pulso carotídeo.

*Asociación significativa entre las variables. 
Tabla 6 Características de las mujeres sin disfunción endotelial previa, basales y posterior al año de observación, según estado actual de la función endotelial

\begin{tabular}{|c|c|c|}
\hline Función endotelial (FMD) & Normal $(>5 \%) n=11$ & Disfunción endotelial $(<5 \%) n=4$ \\
\hline \multicolumn{3}{|l|}{ Variables clínicas } \\
\hline Edad (años) & $53(5,3)$ & $54,5(3,5)$ \\
\hline IMC (Kg/talla m²) & $25,35(7,3)$ & $25,60(13,90)$ \\
\hline $\mathrm{CC}(\mathrm{cm})$ & $84(23)$ & $90,5(28)$ \\
\hline PAS mm Hg) & $120(40)$ & $120(30)$ \\
\hline PAD mm Hg & $75(20)$ & $80(25)$ \\
\hline \multicolumn{3}{|l|}{ Variables hormonales } \\
\hline FSH & $68,18(132,5)$ & $64,90(73,0)$ \\
\hline Estradiol & $30,51(52,5)$ & $32,60(64,30)$ \\
\hline \multicolumn{3}{|l|}{ Variables bioquímicas } \\
\hline Glucemia (mmol/L) & $4,45(1,7)$ & $4,65(1,80)$ \\
\hline Colesterol total (mmol/L) & $4,90(2,0)$ & $5,9(4,6)$ \\
\hline Triglicéridos (mmol/L) & $1,0(1,3)$ & $1,3(3,7)$ \\
\hline
\end{tabular}

CC: circunferencia de cintura; FSH: hormona folículo estimulante; IMC: índice de masa corporal; PAD: presión arterial diastólica; PAS: presión arterial sistólica.

Nótese presencia de riesgo cardiovascular (incremento de la circunferencia de cintura, del colesterol total) en las mujeres que desarrollaron disfunción endotelial evolutivamente.

evolutivo es disfunción endotelial, esclerosis arterial, placa de ateroma, obstrucción vascular, inestabilidad de la placa, con trombosis/embolia y oclusión ${ }^{7,8,13,14}$.

La esclerosis arterial se identifica desde el punto de vista clínico, con los niveles de tensión arterial y la presión del pulso, y se confirma mediante diferentes técnicas ecocardiográficas que identifican la rigidez arterial, que en esta investigación se obtuvo mediante los parámetros de VLPP (el que con mayor precisión la identifica), la elastancia y la compliance, y la obstrucción de la luz arterial por la placa de ateroma previo engrosamiento de la íntima (identificados en esta investigación mediante el grosor íntima media de las arterias carótidas). Según los resultados de nuestra investigación, aunque sin diferencias estadísticas, en las mujeres con daño endotelial se encontraron cambios arteriales que sugieren rigidez arterial, sin manifestaciones compatibles con obstrucción de la luz arterial (ateromas).

Estos resultados se consideran equiparables o similares a los reportados por Thurston et al. ${ }^{25}$ en el estudio retrospectivo SWAN (Study of Women Health Across the Nation) realizado en los Estados Unidos de América, que mostró que a medida que la mujer transita por el climaterio se incrementa la posibilidad de desarrollar aterosclerosis ${ }^{25}$, así como a los de Casiglia et al. $^{26}$ que en 1.031 mujeres con un rango de edad entre 18-95 años, encontraron un incremento significativo de la VLPP en mujeres posmenopáusicas respecto a mujeres premenopáusicas, relación que desapareció después del ajuste por edad, o a los de Nagai et al. ${ }^{27}$ que evaluaron la influencia de la edad y la terapia de reemplazo hormonal con estrógenos en la rigidez arterial carotídea y reportaron que el índice de rigidez arterial fue significativamente menor en las mujeres postmenopáusicas usuarias de la terapia hormonal incluso después del ajuste por la edad, y finalmente a los de Shulfel et al. ${ }^{28}$ en 161 mujeres con edades entre 42 y 61 años sin enfermedad cardiovascular en las que calcularon el índice de distensibilidad basal y tres años después reportaron en las $51 / 161$ que recibían terapia hormonal para la menopausia, mayor compliance arterial que en las no usuarias.

Los resultados descritos justifican en la práctica la asociación entre estrógenos y compliance arterial; sin embargo, no explican por qué la disfunción endotelial no siempre progresa a la esclerosis vascular, y por tanto dificultan identificar su valor pronóstico, es decir, quiénes desarrollarán aterosclerosis. Al respecto, Muller et al. ${ }^{29}$ en mujeres con menopausia reciente con bajo riesgo cardiovascular seguidas durante tres años, también reportan presencia de disfunción endotelial y de grosor íntima media de las arterias carótidas y determinan diferentes sustancias (microvesículas) que proceden del endotelio y de células inflamatorias, las que se relacionan con incremento del grosor íntima media de las arterias carótidas, pero con la disfunción endotelial. Los autores consideran que, si bien las mismas pudieran ser el puente de unión entre daño endotelial y desarrollo de aterosclerosis, aun no es posible evaluar la utilidad pronóstica de las mismas en mujeres asintomáticas. Si se tienen en cuenta aquellos resultados que muestran que en las mujeres con disfunción endotelial la mayor intensidad de los síntomas vasomotores aumenta el riesgo para incrementar la velocidad de propagación del pulso (parámetro que mejor representa la rigidez arterial), sería posible proponer una nueva visión del síntoma vasomotor, pues además de ser la manifestación típica de la transición a la menopausia, se convertiría en un probable "marcador" de riesgo vascular.

La grasa epicárdica, de manera similar a lo que ocurre con la grasa visceral abdominal, se relaciona con factores como la resistencia a la insulina, la dislipidemia y el síndrome metabólico, entre otros ${ }^{18-20,30}$, por lo que se puede presumir desde el punto de vista fisiológico, que ambas tengan una respuesta similar a los cambios en el patrón hormonal de la mujer en etapa de climaterio; sin embargo, hasta el presente no se dispone de reportes que permitan confirmar dicha suposición. En este estudio, en las mujeres 
con disfunción endotelial se encontró correlación positiva entre el grosor de la grasa epicárdica con el índice de masa corporal pero no con la circunferencia de cintura, y por otra parte en las mujeres que evolutivamente desarrollaron disfunción endotelial hubo incremento de peso, de la circunferencia de cintura y de la grasa epicárdica, resultados no concluyentes y de difícil explicación patogénica, que plantean la necesidad de realizar investigaciones para identificar los cambios en la grasa epicárdica durante el climaterio y su utilidad pronóstica.

El subgrupo (aunque pequeño) de mujeres en que se diagnosticó disfunción endotelial evolutivamente, también presentó incremento de la circunferencia de cintura, así como del colesterol total y de la glucemia en ayunas (factores de riesgo para aterosclerosis) sin modificación en la tensión arterial ni en los niveles de estradiol, al compararlos con la evaluación inicial; por tanto, es válido considerarlos, no como expresión del momento biológico por el que transita la mujer, sino probablemente de un estilo de vida no saludable, lo que confirma la multitud de factores capaces de afectar al endotelio y que mientras no se disponga de los métodos diagnósticos más específicos para identificar personas que progresen o desarrollen la aterosclerosis, la mejor arma para su prevención está en la educación para la salud.

Finalmente, con base en estos resultados y lo reportado por otros investigadores, en tanto no se cuente con métodos más específicos, consideramos pertinente plantear que ante toda mujer en etapa de climaterio que refiera síntomas vasomotores de intensidad "molesta" aun con bajo riesgo cardiovascular se debería evaluar la función endotelial, de ocurrir disfunción realizar búsqueda activa de manifestaciones de rigidez arterial, instituir las medidas terapéuticas correspondientes para el control de los síntomas y mantener observación durante el mayor periodo de tiempo posible, sin olvidar reforzar la necesidad de lograr y mantener un estilo de vida saludable aun en ausencia de daño endotelial.

\section{Limitaciones del estudio}

El sesgo derivado del poco tiempo (1 año) de observación y del pequeño número de mujeres reevaluadas.

\section{Financiación}

Ninguna.

\section{Conflicto de intereses}

Ninguno.

\section{Agradecimientos}

A las mujeres que participaron en la investigación.

\section{Bibliografía}

1. Schenck-Gustafsson K, Brincat M, Tamer C, Gambacciani M, Lambrinoudaki I, Moen MH, et al. EMAS position statement: Managing the menopause in the context of coronary heart disease. Maturitas. 2011;68:94-7.
2. Santoro N. Perimenopause: From Research to Practice. J Womens Health (Larchmt). 2016;25:332-9, http://dx. doi.org/10.1089/jwh.2015.5556. Epub 2015 Dec 10.

3. Barret-Connor E. Menopause, atherosclerosis and coronary artery disease. Curr Opin Pharmacol. 2013;13:186-91.

4. Kunstmann S, de Grazia R, Gainza D. Aterosclerosis en la mujer: factores de riesgo y prevención. Rev Chil Cardiol. 2012;31:142-7.

5. Thurston RC, El Khoudary SR, Sutton-Tyrrell K, Crandall CJ, Gold EB, Sternfeld B, et al. Vasomotor symptoms and lipid profiles in women transitioning through menopause. Obstet Gynecol. 2012;119:753-61.

6. Mulvagh SL, Behrenbeck T, Lahr BA, Bailey KR, Zais TG, Araoz PA, Miller VM. Endothelial function and cardiovascular risk stratification in menopausal women. Climacteric. 2010;13:45-54, http://dx.doi.org/10.3109/13697130902943287.

7. Peters SA, den Ruijter HM, Bots ML, Moons KG. Improvements in risk stratification for the occurrence of cardiovascular disease by imaging subclinical atherosclerosis: a systematic review. Heart. 2012;98:177-84, http://dx. doi.org/10.1136/heartjnl-2011-300747. Epub 2011 Nov 17.

8. Yeboah J, McCleveland RL, Polonsky TS, Burke GL, Sibley ChT, O'Leary D, et al. Comparison of novel risk markers for improvement in cardiovascular risk assessment in intermediate risk individuals. The Multi-Ethnic Study of Atherosclerosis. JAMA. 2012;308:788-95, http://dx.doi.org/10.1001/jama.2012.9624.

9. Llerena-Rojas LR, Peix-González A, Valiente-Mustelier J. Técnicas de imagen no invasivas en la valoración y la prevención de la enfermedad coronaria. Rev Cubana Cardiol Cir Cardiovasc. 2011;17 Supl1:47-57.

10. Muscelli E, Kozàkovà M, Flyvbjerg A, Kyriakopoulou K, Astiarraga BD, Glintborg D, et al. The effect of menopause on carotid artery remodeling, insulin sensitivity, and plasma adiponectin in healthy women. Am J Hypertens. 2009;22:364-70.

11. Bechlioulis A, Kalantaridou SN, Naka KK, Chatzikyriakidou A, Calis KA, Makrigiannakis A, et al. Endothelial function, but not carotid intima-media thickness, is affected early in menopause and is associated with severity of hot flushes. J Clin Endocrinol Metab. 2010;95:1199-206.

12. Wang KL, Cheng HM, Sung SH, Chuang SY, Li CH, Spurgeon HA, et al. Wave reflection and arterial stiffness in the prediction of 15-year all-cause and cardiovascular mortalities: a communitybased study. Hypertension. 2010;55:799-805.

13. Shufelt Ch, Elboudwarej $O$, Johnson BD, Mehta P, Bittner V, Braunstein G, et al. Carotid artery distensibility and hormone therapy and menopause: The Los Angeles Atherosclerosis Study (LAAS). Menopause. 2016;23:150-7, http://dx.doi.org/10.1097/GME. 0000000000000510.

14. El Khoudary SR, Wildman RP, Matthews K, Thurston RC, Bromberger JT, Sutton-Tyrrell K. Progression rates of carotid intima-media thickness and adventitial diameter during the menopausal transition. Menopause. 2013;20:8-14, http://dx.doi.org/10.1097/gme.0b013e3182611787.

15. Wolff EF, He Y, Black DM, Brinton EA, FAHA, FNLA, Budoff MJ, et al. Self-reported menopausal symptoms, coronary artery calcification and carotid intima-media thickness in recently menopausal women screened for the Kronos Early Estrogen Prevention Study (KEEPS) Fertil Steril. 2013;99:1385-91, http://dx.doi.org/10.1016/j.fertnstert.2012.11.053.

16. Cabrera-Rego JO, Gandarilla-Sarmientos JC, Del Busto-Mesa A, Valiente-Mustelier J. Association between epicardial fat, HOMA-IR and carotid intima-media thickness. Rev Arg Cardiol. 2012;80:222-9.

17. Cabrera-Rego JO, Gandarilla JC, Valiente J, Olivares EW, Mendoza V, Armas N. Grasa epicárdica y su asociación con aterosclerosis subclínica y otros factores de riesgo en pacientes con síndrome metabólico. Clin Invest Arterioscl. 2011;23: 245-52. 
18. Cabrera-Rego JO, lacobellis G, Sarmientos JC, Mustelier JV, Aquiles EW, Rodriguez VM, et al. Epicardial fat correlates with $\mathrm{ApoB} / \mathrm{ApoA} 1$ ratio, coronary calcium and carotid intima media thickness in asymptomatic patients. Int $\mathrm{J}$ Cardiol. 2011;151:234-6.

19. Navarro D, Duany A. Expresión del cese de la función reproductiva del ovario. En: Artiles L., Manzano BM., Navarrro D (Eds): Climaterio y Menopausia. Un enfoque desde la social. La Habana, Editorial Científico Técnica, La Habana 2007. p. 11-22.

20. Sariol Y, Navarro D, Álvarez R, Armas Y, Domínguez E, Dopazo M. Etapas del climaterio y función endotelial en mujeres de edad mediana. Rev Cubana Endocrinol. 2015;26:138-46.

21. Pérez Piñero J, Martínez Morales MA, Sarmiento Leyva M. Escala climatérica como instrumento para la clasificación de la severidad del síndrome climatérico. En: Artiles Visbal L, Navarro Despaigne D, Manzano Ovies BR, editores. Climaterio y menopausia. Un enfoque desde lo social. La Habana: Editorial Científico Técnica; 2007. p. 501-12.

22. Institute of Isotopes Ltd. 1535 Budapest Pf.: 851.

23. TzouWS, Douglas PS, Srinivasan SR, Bond MG, Tang R, Chen W, et al. Distribution and predictors of carotid artery intimamedia thickness in young adults: the Bogalusa Heart Study. Prev Cardiol. 2007; 10:181-9.

24. Valiente J, Suárez L, Cabrera JO, Gandarilla JC. Evaluación ecocardiográfica de la rigidez arterial en sanos e hipertensos menores de 60 años. Rev Cubana Cardiol Cir Cardiovasc. 2011;17:234-41
25. Thurston RC, Khoudary SR, Tepper PG, Jackson EA, Joffe H, Chen H-Y, Mathews KA. Trajectories of vasomotor symptoms and carotide intima media thickness in the study of Women's Health Across the Nation. Stroke J. 2016;47:12-7.

26. Casiglia E, Tikhonoff V, Boschetti G, Giordano N, Mazza A, Caffi S, Palatini P. Arterial stiffness and related variables across menopausal status: An Epidemiologic Study. J Women's Health. 2013;22:75-84

27. Nagai Y, Earley CJ, Kemper MK, Bacal CS, Metter EJ. Influence of age and postmenopausal estrogen replacement therapy on carotid arterial stiffness in women. Cardiovasc Res. 1999;41:307-11.

28. Shufelt Ch, Elboudwarej $\mathrm{O}$, Johnson BD, Mehta P, Bittner V, Braunstein G, et al. Carotid artery distensibility and hormone therapy and menopause: The Los Angeles Atherosclerosis Study (LAAS). Menopause. 2016;23:150-7, http://dx.doi.org/10.1097/GME.0000000000000510.

29. Miller VM, Lahr BD, Bailey KR, Hodis HN, Mulvagh SH, Jayachandran $M$. Specific cell-derived microvesicles: linking endothelial function to carotid artery intima-media thickness in low cardiovascular risk menopausal women. Atherosclerosis. 2016;246:21-8, http://dx.doi.org/10.1016/ j.atherosclerosis.2015.12.030.

30. Cabrera E. Evaluación ecocardiográfica de la grasa epicardica como marcador de riesgo cardiometabólico en pacientes cubanos. Tesis para optar por el grado científico de Doctor en Ciencias Médicas. Universidad de Ciencias Médicas de La Habana. Hospital Dr. "Miguel Enríquez". Instituto de Cardiología y Cirugía Cardiovascular. 2013. 